\title{
Erfahrungen mit der Krankenhausplanung in Nordeuropa
}

UWE K. PREUSKER

Dr. Uwe K. Preusker ist Vorstand der Preusker Health Care OY und lebt in Vantaa/Finnland

Die Krankenhausplanung oder genauer die Kapazitätsplanung für die ambulante und stationäre fachärztliche und die Notfallversorgung sowie die hierfür erforderliche Finanzierung der Investitionen ist in den nordeuropäischen Gesundheitssystemen überwiegend Aufgabe der für die gesundheitliche Versorgung und deren Finanzierung zuständigen regionalen/kommunalen Gebietskörperschaften. Hochspezialisierte Versorgungsbereiche werden dagegen in ausgesuchten Zentren konzentriert. Meist liegen diesen Entscheidungen konsensuale Prozesse zugrunde. Die Zentralstaaten versuchen schrittweise Planungsfunktionen stärker zu konzentrieren, vor allem durch zweckgebundene Zuschüsse zu Investitionsvorhaben im Krankenhausbereich.

\section{Geographische Ausgangslage}

Eine Nord-Süd-Ausdehnung von rund 1.500 Kilometern, eine durchschnittliche Bevölkerungsdichte zwischen 14 und 20 Einwohnern pro Quadratkilometer - das sind die Eckwerte, mit denen sich Finnland, Norwegen und Schweden in Sachen Gesundheitsversorgung und damit auch der Krankenhausplanung auseinandersetzen müssen. Dabei stellt sich die Situation in den nördlichen Regionen dieser drei Länder nochmals deutlich schwieriger dar: Dort gibt es zum Teil nur zwei Einwohner pro Quadratkilometer. Ein Beispiel illustriert, was Gesundheitsversorgung unter solchen Bedingungen bedeutet: Die weiteste Entfernung, die eine werdende Mutter in Finnland zurücklegen muss, um in die nächste Geburtsklinik zu kommen, beträgt rund 550 Kilometer - von der nördlichen Landesgrenze zu Norwegen bis hinunter zum Polarkreis nach Rovaniemi. Im dortigen Zentralkrankenhaus gibt es die einzige geburtshilfliche Abteilung im weiten Umkreis. Die Situation in Nordschweden oder Nordnorwegen ist ähnlich - die Verhältnisse unterscheiden sich nur graduell.

Eine andere Situation weist dagegen Dänemark auf, der vierte und südlichste nordeuropäische Flächenstaat: Die Bevölkerungsdichte in Dänemark betrug im Jahr 2011 129,47 Einwohner pro Quadratkilometer, und die Fläche, die Dänemark einnimmt, beläuft sich nur auf 43.000 qkm, während die anderen drei Flächenstaaten eine Flächenausdehnung zwischen 338.000 und 450.000 $\mathrm{qkm}$ aufweisen (siehe Tabelle 1). 
Tabelle 1: Geografische Daten der nordeuropäischen Staaten im Überblick

\begin{tabular}{|l|c|c|c|}
\hline Land & Fläche in qkm & $\begin{array}{c}\text { Einwohnerzahl in } \\
\text { Millionen }\end{array}$ & $\begin{array}{c}\text { Bevölkerungsdichte } \\
\text { pro qkm }\end{array}$ \\
\hline Deutschland & 357.021 & 82,3 & 231 \\
\hline Dänemark & 43.000 & 5,56 & 129 \\
\hline Finnland & 338.145 & 5,29 & 16 \\
\hline Norwegen & 386.958 & 4,66 & 15 \\
\hline Schweden & 449.964 & 9,06 & 21 \\
\hline
\end{tabular}

Quelle. Länder-Informationen, eigene Recherchen

\section{Organisation und Finanzierung der Gesundheitsversorgung: Aufgabe von Kommunen und Regionen}

Organisation und Finanzierung der gesundheitlichen Versorgung in Nordeuropa unterscheiden sich in einigen zentralen Aspekten vom deutschen Modell. Die Versicherungspflicht beruht in allen nordeuropäischen Ländern auf dem Wohnsitzprinzip. Sie umfasst damit jeden im Lande gemeldeten Einwohner. Damit ist in Nordeuropa grundsätzlich jede im Lande gemeldete Person krankenversichert, unabhängig von ihrer Nationalität, einer Berufstätigkeit oder

\subsection{Sicherstellungsauftrag}

Der Sicherstellungsauftrag für die gesundheitliche Versorgung der Bevölkerung liegt in allen vier nordeuropäischen Gesundheitssystemen bei Gebietskörperschaften, die unterhalb der gesamtstaatlichen Ebene angesiedelt zusammenschlüsse beziehungsweise Provinziallandtage oder Regionen). In bietskörperschaften mit demokratisch ihrem Einkommen. sind (Kommunen oder Kommunalallen Fällen handelt es sich dabei um Ge-

leisten die Versorgung im Normalfall durch Einrichtungen, deren Träger sie auch sind. Die Privatisierung von Gesundheitseinrichtungen und speziell Krankenhäusern ist dagegen in Nordeuropa bisher eher selten. Zwar gibt es einige Beispiele, in denen der Betrieb von Krankenhäusern von privaten Trägern übernommen worden ist - so etwa der Betrieb des St. Göran-Krankenhauses in Stockholm, dem bisher einzigen privatisierten schwedischen Akutkrankenhaus -, doch Gebäude sowie Grund und Boden bleiben auch in solchen Fällen im Eigentum des öffentlichen Trägers. Auch werden Versorgungsverträge mit privaten Betreibern nur für relativ kurze Zeitspannen abgeschlossen. So sind Laufzeiten von drei Jahren mit einer Verlängerungsmöglichkeit um ein bis zwei Jahre durchaus üblich. Dagegen werden mittlerweile zunehmend häufig Gesundheitsleistungen überregional ausgeschrieben und damit ein Preis- und Qualitätswettbewerb ausgelöst.

\subsection{Wahlfreiheit und Behandlungsgarantie}

Mit dem Prinzip der Sicherstellung und der Eigentümerschaft der Gesundheitseinrichtungen durch die Gebietskörperschaften eng verbunden war lange Zeit die Beschränkung der Wahlfreiheit: Der Bürger konnte im Prinzip medizinische Behandlung nur in den Einrichtungen der eigenen, für die gesundheitliche Versorgung zuständigen $\mathrm{Ge}$ -

auf Zeit gewählten Parlamenten und Führungsgremien und damit mit direkter demokratischer Kontrolle und Legitimation. Der Versorgungsauftrag ist entsprechend dieser Grundkonstruktion auf die Wohnbevölkerung der jeweiligen Gebietskörperschaft beschränkt. Die Gebietskörperschaften gewähr- bietskörperschaft nachsuchen. Dieses Grundprinzip des Zusammenfallens von Versorgungsauftrag und Leistungserbringung hat sich jedoch seit nunmehr rund 20 Jahren zunehmend verändert: Immer häufiger werden Leistungen gebietsüberschreitend ausgeschrieben, wobei auch private Leistungserbringer zugelassen werden. Gleichzeitig hat die Wahlfreiheit der Bürger deutlich zugenommen, einerseits durch so genannte Behandlungsgarantien, die durch jüngere Reformmaßnahmen in allen nordeuropäischen Gesundheitssystemen eingeführt wurden, andererseits durch die gesetzlich oder per Vereinbarungen des Zentralstaates mit den Gebietskörperschaften gewährleistete Wahlfreiheit für den Ort der Behandlung. In Dänemark ist diese Wahlfreiheit zum Beispiel zu Beginn des Jahres 1993 in Kraft getreten, und seit Mitte 2002 gilt eine Behandlungsgarantie, die dem einzelnen Patienten die Möglichkeit gibt, sich auch in privaten oder ausländischen Krankenhäusern, die Verträge mit der Vereinigung der dänischen Regionen haben, behandeln zu lassen, wenn die vorgeschriebene Höchst-Wartezeit von einem Monat überschritten wird.

\subsection{Finanzierung}

Die Finanzierung der Gesundheitssysteme in Finnland, Norwegen und Schweden erfolgt weit überwiegend über Steuern. Dabei gibt es eine hohe Übereinstimmung der finanziellen und gestalterischen Verantwortung: Die Gebietskörperschaft, die für die Sicherstellung und Erbringung der gesundheitlichen Versorgung zuständig ist (durch eigene Einrichtungen oder durch den Abschluss von Verträgen mit anderen, auch privaten Einrichtungen), hat auch das Recht zur Erhebung von Steuern, mit denen ein großer Teil der Gesundheitsausgaben auf der Ebene der einzelnen Gebietskörperschaft gedeckt wird. Zusätzlich zu diesen eigenen Steuern gibt es Zuschüsse vom Zentralstaat - am stärksten ausgeprägt in Norwegen. So haben zum Beispiel in Finnland die knapp 350 Kommunen sowohl den Sicherstellungsauftrag für die gesundheitliche Versorgung als auch das Recht zur Erhebung einer eigenen linearen Einkommensteuer, aus der die Ausgaben der Kommune auch für gesundheitliche Dienstleistungen - gedeckt werden und die derzeit je nach Kommune zwischen 18 und 22 Prozent vom steuerbaren Einkommen beträgt. In Schweden dagegen sind es die 18 Provinziallandtage (landsting) und zwei Regionen, die die direkte $\mathrm{Zu}$ ständigkeit für die Sicherstellung und Versorgung mit Gesundheitsleistungen haben. Auch sie haben das Recht, eine 
eigene Einkommensteuer $\mathrm{zu}$ erheben, aus der der größere Teil der Ausgaben für die gesundheitliche Versorgung bestritten wird.

Das dänische Gesundheitssystem ${ }^{1}$ bildet bei der Finanzierung der Gesundheitsausgaben seit der grundlegenden Gebietsreform von 2007 eine Ausnahme: Mit dieser Reform, mit der die bis dahin 14 Ämter (Kreise) und 271 Kommunen des Landes in nur noch fünf Regionen und 98 Kommunen aufgegangen sind, wurde die Zuständigkeit für die Finanzierung der Gesundheitsversorgung weitestgehend beim dänischen Staat angesiedelt. Er erhebt seither von allen Bürgern eine achtprozentige Gesundheitssteuer auf das zu versteuernde Einkommen. Aus deren Aufkommen erhalten die für die Sicherstellung und Erbringung von Gesundheitsleistungen zuständigen fünf dänischen Regionen entsprechende Zuweisungen. Sie machen rund 80 Prozent der Gesamtgesundheitskosten der Regionen aus. Der größte Teil dieser staatlichen Zuweisungen (75 Prozent der Gesamtkosten) wird auf der Grundlage von objektiven Faktoren wie Demographie und soziale Struktur der jeweiligen Region ermittelt, die restlichen fünf Prozent werden basierend auf Aktivitätsdaten der jeweiligen Region zugewiesen. 20 Prozent der Gesundheitskosten der Regionen werden von den Kommunen aufgebracht, davon die Hälfte basierend auf InanspruchnahmeDaten durch die jeweilige Wohnbevölkerung der Kommune. Die 98 dänischen Kommunen haben das Recht zur Erhebung einer eigenen Einkommensteuer. Sie sind selbst für die gesamte Altenpflege, Rehabilitation, Prävention, Behandlung von Drogen- und Alkoholabhängigkeit sowie die zahnärztliche Versorgung von geistig Behinderten zuständig.

\subsection{Gebietsreformen zur Schaffung leistungsfähiger Einheiten}

Um die Fähigkeit der Gebietskörperschaften zu verbessern, die gesundheitliche Versorgung sowie auch andere Aufgaben vor allem im Sozialbereich effizient zu erledigen, gibt es in den nordeuropäischen Gesundheitssystemen seit einiger Zeit das Bemühen, den Zuschnitt der Gebietskörperschaften, in ihrer Grö- ße so leistungsfähig zu gestalten, dass sie den wachsenden Anforderungen durch die ständig zunehmenden medizinischtechnischen Möglichkeiten einerseits und die zunehmende Alterung der Bevölkerung andererseits gerecht werden. Hier ist neben Dänemark auch Finnland aktiv, das den Zusammenschluss vor allem kleinerer Kommunen zu leistungsfähigeren Einheiten systematisch und gezielt fördert - ein Prozess, der noch nicht abgeschlossen ist.

\section{Dreistufiger Aufbau der Versorgungsstruktur}

Eine Gemeinsamkeit der nordeuropäischen Gesundheitssysteme ist die von Deutschland unterschiedliche Gliederung der Versorgung in ambulante Primärversorgung einerseits sowie fachärztliche ambulante und stationäre Versorgung, vielfach durch die gleichen Ärzte überwiegend an Kliniken, andererseits. Damit wird die deutsche Besonderheit der doppelten Vorhaltung einer spezialärztlichen ambulanten und stationären Versorgungsstruktur weitgehend vermieden. Damit einher geht auch eine andere Aufgabenteilung zwi-

\section{Die deutsche Besonderheit} der doppelten Vorhaltung einer spezialärztlichen ambulanten und stationären

\section{Versorgungsstruktur wird} weitgehend vermieden.

schen den Gesundheitsberufen, insbesondere zwischen dem ärztlichen und pflegerischen Berufsstand: Pflegekräfte haben deutlich weitergehendere Kompetenzen und Befugnisse, die bis zur Verordnung bestimmter Arzneimittel durch entsprechend erfahrene und qualifizierte Pflegepersonen reichen. Ein weiteres Beispiel: Hausbesuche durch Ärzte sind in Nordeuropa unüblich. Diese Aufgabe der häuslichen Betreuung wird insbesondere in Schweden und Finnland über das System der Bezirkskrankenschwester von Pflegepersonen übernommen.

Die gesundheitliche Versorgung ist dreistufig aufgebaut: Zusätzlich zur ambulanten primärärztlichen Versorgung existiert ein zweistufiges fachärztliches Versorgungssystem an Krankenhäusern.

\subsection{Ambulante primärärztliche Versorgung - Gesundheitszentren}

Die ambulante primärärztliche Versorgung obliegt in Schweden, Finnland und teilweise auch in Norwegen Gesundheitszentren, die sich meist in der Trägerschaft von Kommunen befinden. Insbesondere in dünn besiedelten Regionen ist dies häufig der einzige Weg, die Versorgung sicherzustellen, da eine ausreichende gesundheitliche Versorgung durch in eigener Praxis niedergelassene Ärzte gerade in dünn besiedelten Gebieten nicht denkbar wäre.

In Dänemark dagegen wird die ambulante primärärztliche Versorgung von rund 4.100 in eigener Praxis niedergelassenen Allgemeinärzten (Hausärzten) gewährleistet, die im Durchschnitt jeweils rund 1.300 Patienten zu versorgen haben. Die ambulante fachärztliche Versorgung findet überwiegend in Kliniken und ergänzend durch rund 1.200 in eigener Praxis niedergelassene Fachärzte statt. Sowohl die in eigener Praxis niedergelassenen Allgemein- als auch Fachärzte haben mit den Regionen Versorgungsverträge abgeschlossen. Die Vergütungen werden kollektiv zwischen der Vereinigung der dänischen Regionen und den Organisationen der Ärzte abgeschlossen. Vergütungsverträge bedürfen allerdings zu ihrem Wirksamwerden der Zustimmung des dänischen Gesundheitsministers. Norwegen dagegen verfügt über ein Mischsystem: Vor allem in der Region Oslo und den übrigen größeren Städten wird die Primärversorgung - ähnlich wie in Dänemark - durch niedergelassene Ärzte sichergestellt. In den westlichen und nördlichen Teilen Norwegens und in kleineren Städten und Gemeinden dagegen erfolgt die Primärversorgung durch bei den Kommunen angestellte Ärzte (Kommunelege) in den kommunalen Gesundheitszentren.

In den Gesundheitszentren bzw. Arztpraxen wird sowohl die Akutversorgung der Bevölkerung in leichten und mittelschweren Fällen übernommen als auch die laufende Versorgung von chronisch Kranken sichergestellt. Dabei arbeiten in den Gesundheitszentren typischer Weise Allgemeinärzte, Internisten und

1 Vgl. auch: Ministry of the Interior and Health, Health Care in Denmark, Kopenhagen 2008 
Kinderärzte, in selteneren Fällen auch weitere Fachrichtungen. Darüber hinaus sind die Gesundheitszentren mit Pflegepersonal besetzt, das zum Teil auch Spezialisierung vor allem zur Versorgung chronisch Kranker aufweist (z.B. Diabetes- oder Herz-Kreislauf-Schwestern). In den Gesundheitszentren werden auch Vorsorgemaßnahmen sowie Impfungen vorgenommen. Außerdem findet hier ambulante Rehabilitation statt. In vielen Kommunen sind die Gesundheitszentren außerdem auch für Präventionsangebote zuständig. Die gesundheitliche Betreuung von Säuglingen und Kindern dagegen erfolgt durch spezialisiertes Personal, das meist in Räumen arbeitet, die an Kindergärten oder Schulen angeschlossen sind. Dort sind diese Beratungsangebote für die Eltern leicht zu erreichen; die Nutzung ist in allen nordeuropäischen Staaten außerordentlich hoch. Vor allem in den dünner besiedelten Gebieten im

\section{Die ambulante und stationäre fachärztliche Betreuung übernehmen in allen nordeuropäischen Ländern Krankenhäuser.}

Norden Nordeuropas gehört zu den Gesundheitszentren im Normalfall eine kleine Betten-Abteilung, die vor allem für solche Erkrankungsfälle vorgesehen ist, die zur Beobachtung bleiben müssen, aber nicht unmittelbar zu spezialisierten Weiterbehandlung an ein Krankenhaus überwiesen werden müssen. Immer wieder werden diese Betten aber auch genutzt, um Patienten vorübergehend unterzubringen, die aus der stationären Behandlung kommen und für die es noch keinen Pflegeplatz gibt.

In allen nordeuropäischen Gesundheitssystemen existiert heute ein Hausbeziehungsweise Primärarzt-System. Das stringenteste Primärarztsystem haben Dänemark, Finnland und Norwegen, wo das Aufsuchen der fachärztlichen Ambulanz oder auch des niedergelassenen Facharztes - bis auf akute Ausnahmefälle - nur mit Überweisung eines Primärarztes möglich ist. In Schweden dagegen können fachärztliche Ambulanzen auch direkt aufgesucht werden das Hausarztsystem ist hier als Angebot ausgestaltet; die Steuerung geschieht vor allem über entsprechend ausgestaltete
Selbstbeteiligungs-Regelungen. Ein zentraler Unterschied zu Deutschland ist die relativ geringe Zahl der Arztkontakte (ambulante Behandlung bei Haus- und Fachärzten) pro Kopf der Bevölkerung in den nordeuropäischen Ländern. Nach der OECD-Statistik gab es 2010 in Schweden lediglich 2,9 Arztkontakte pro Kopf der Bevölkerung, während Deutschland hier mit einem Wert von 8,9 Arztkontakten pro Kopf der Bevölkerung aufgeführt wird (Finnland: 4,3; Dänemark: 4,6; Norwegen (2009): 5,2 Arztkontakte pro Kopf der Bevölkerung) $)^{2}$.

\subsection{Krankenhäuser}

Die ambulante und stationäre fachärztliche Betreuung übernehmen in allen nordeuropäischen Ländern Krankenhäuser. In eigener Praxis niedergelassene Fachärzte existieren; ihr Anteil an der Gesamtversorgung ist aber eher gering. Ebenfalls an den Akutkrankenhäusern angesiedelt ist die Notfallversorgung in schwereren Fällen.

In Schweden befinden sich die Krankenhäuser fast ausschließlich in der Trägerschaft der Provinziallandtage bzw. Regionen. Bei den Kliniken in der Trägerschaft der Provinziallandtage/ Regionen wird zwischen Akutkrankenhäusern und lokalen Krankenhäusern, die keine Akutbehandlung erbringen, unterschieden. Knapp 80 Prozent der Krankenhäuser sind Akutkliniken. ${ }^{3}$

In Finnland dagegen müssen alle Kommunen per Gesetz Mitglied in einem von insgesamt 20 Krankenhausbezirken sein. Diese Krankenhausbezirke sind dann Träger der Krankenhäuser im jeweiligen Bezirk. Die Mitglieds-Kommunen des Krankenhausbezirks beziehen die benötigten fachärztlichen Leistungen vom Krankenhausbezirk, können aber auch über Ausschreibungen von anderen Krankenhausbezirken oder von privaten Kliniken Leistungen beziehen. Bei den Verhandlungen über Preis und Menge der benötigten Leistungen spielt der (im Normalfall ärztliche) Leiter der Gesundheitsversorgung in einer Kommune eine zentrale Rolle. Er ist nicht nur verantwortlich für die Gesundheitszentren der Kommune, sondern auch für den Einkauf der benötigten spezialärztlichen Leistungen.
Die Anfang 2002 in Norwegen in Kraft getretene Krankenhausreform hat zu einer Zentralisierung der Klinikträgerschaft beim norwegischen Staat geführt. Alle in öffentlicher Trägerschaft befindlichen Kliniken sowie weitere spezialisierte Gesundheitseinrichtungen wurden dazu fünf Gesundheitsregionen zugeordnet, die in der Rechtsform einer nicht börsennotierten öffentlichen Aktiengesellschaft geführt werden. Inhaber ist zu 100 Prozent der norwegische Staat; die Hauptversammlung aller fünf Gesundheits-AGs stellt der jeweils amtierende norwegische Gesundheitsminister dar. Auch in Norwegen ist es so, dass die Kommunen beziehungsweise die größeren Fylkekommunen die benötigten Mengen an Leistungen mit dem für die Region zuständigen Krankenhausunternehmen verhandeln. Die Finanzierung der Krankenhäuser geschieht in Norwegen über ein System, bei dem neben staatlichen Budget-Zuweisungen für etwa 40 Prozent der Kosten die jeweilige Wohngemeinde für die einzelne Behandlung eine Fallpauschale nach dem nordischen DRG-System bezahlen muss.

In Dänemark ist zum 1. Januar 2007 eine Regionalreform in Kraft getreten, mit der die bis dahin 14 Ämter (Kreise) und 271 Kommunen des Landes in nur noch fünf Regionen und 98 Kommunen aufgegangen sind. Mit dieser Organisationsreform ging eine Stärkung der $\mathrm{Zu}$ ständigkeiten der vergrößerten Ämter und Kommunen einher. Ziel der Reform war vor allem eine Stärkung der Kommunen und Regionen in ihrer Fähigkeit, Aufgaben für die Wohnbevölkerung darunter auch diejenigen im Gesundheitssystem - selbst wahrnehmen und finanzieren zu können. Die früheren Ämter (den deutschen Landkreisen vergleichbar) wurden im Zuge der Reform abgeschafft. An ihre Stelle traten fünf Regionen mit einer Einwohnerzahl zwischen 0,6 und 1,6 Millionen Einwohnern. Bei ihnen ist die verpflichtende öffentliche dänische Krankenversicherung angesiedelt, in der alle Bewohner des Landes automatisch versichert sind. Darüber hinaus sind sie auch Träger der öf-

2 OECD Health Data 2012 (http://stats.oecd. org)

3 Vgl. Anders Anell, Anna H. Glenngård, Sherry Merkur: Health Systems in Transition Sweden Health System Review; Kopenhagen 2012; S. 90 
fentlichen Krankenhäuser in Dänemark. Aufgabe des dänischen Zentralstaates im Gesundheitssektor ist dagegen die Weiterentwicklung des Rechtsrahmens sowie die allgemeine Planung.

An den meisten regionalen Krankenhäusern in Nordeuropa existieren ambulante Bereitschafts- oder Notaufnahmeeinrichtungen mit 24-Stunden Bereitschaft, in denen Patienten behandelt werden, die wegen der Schwere ihrer Erkrankung oder Verletzung nicht in den Gesundheitszentren behandelt werden können. Sie arbeiten nach dem Prinzip der Triage, wobei für die Triage der Patienten speziell fortgebildetes und langjährig erfahrenes Krankenpflegepersonal zuständig ist. Auf der Grundlage dieser Ersteinschätzung werden die Patienten dann zur eigentlichen ambulanten oder stationären Behandlung an die zuständigen Ärzte direkt in der Bereitschaftseinrichtung oder auch in den Fachabteilungen weitergeleitet.

\subsection{Universitäts- und Schwerpunktkliniken}

\section{Die Einwirkungsmöglichkeiten der Zentralstaaten beziehen sich vor allem auf die Investitionsfinanzierung sowie die Kapazitätsplanung für hochspezialisierte Versorgungsbereiche.}

von 1,79 Betten pro 1.000 Einwohner aufweist. Die durchschnittliche AkutVerweildauer ist mit 3,6 Tagen (2010) in Dänemark am niedrigsten (Deutschland: 7,3 Tage). In allen nordeuropäischen Staaten ist es seit rund 15 Jahren erklärtes Ziel der Gesundheitspolitik, die ursprünglich sehr krankenhauslastigen Versorgungsstrukturen zugunsten von mehr ambulanten Versorgungsange-

Tabelle 2: Krankenhausdichte und Verweildauer im Vergleich (2007)

\begin{tabular}{|l|c|c|}
\hline Land & $\begin{array}{c}\text { Akut-Krankenhaus-betten } \\
\text { pro 1.000 Einwohner 2010 }\end{array}$ & $\begin{array}{c}\text { Durchschnittliche Akut- } \\
\text { Verweildauer 2010 }\end{array}$ \\
\hline Deutschland & 5,66 & 7,3 \\
\hline Dänemark & 2.87 & 3,6 \\
\hline Finnland & 1,79 & 5,4 \\
\hline Norwegen & 2,37 & 4,5 \\
\hline Schweden & 2,02 & 4,6 \\
\hline
\end{tabular}

Quelle: OQECD Health Data 2012

Zusätzlich zu den regionalen Krankenhäusern existieren in allen vier Ländern Universitätskliniken bzw. Regionalkrankenhäuser, die sowohl für die Ausbildung und einen großen Teil der Spezialisierung der Ärzte und weiterer Gesundheitsberufe als auch für die Versorgung besonders seltener und schwieriger Erkrankungen und Verletzungen zuständig sind. In Schweden ist man mittlerweile dazu übergegangen, bestimmte hochspezialisierte Leistungen auf der Grundlage eines Auswahlprozesses nur einem Teil der Hochschulkliniken befristet auf einige Jahre zu übertragen.

Insgesamt liegt sowohl die Kranken- boten umzugestalten. Dazu gehört auch der massive Ausbau der Möglichkeiten für ambulante Operationen. Heute finden sich an allen größeren Klinik-Standorten gesonderte Einrichtungen speziell für das Ausführen von ambulanten Operationen. Dementsprechend ist die Zahl der ambulanten Operationen zu Lasten der stationären Eingriffe auch Jahr für Jahr deutlich gestiegen.

\section{Krankenhausplanung}

Die Krankenhausplanung ist in den nordeuropäischen Gesundheitssystemen Aufgabe der für die Gesundheitsversorgung überwiegend zuständigen regiona- len Gebietskörperschaften (Schweden: Landsting bzw. Provinziallandtage; Finnland: Krankenhausbezirke als Gemeinschaftseinrichtung der zugehörigen Kommunen; Dänemark: Regionen) bzw. in Norwegen Aufgabe der fünf regionalen Gesundheitsunternehmen, bei denen die Trägerschaft der Krankenhäuser des Landes konzentriert sind. Dabei wird im Allgemeinen von der Kapazitätsplanung für die ambulante und stationäre fachärztliche Versorgung sowie die Notfallversorgung gesprochen; ergänzend daneben steht die Investitionsfinanzierung. Die Einwirkungsmöglichkeiten der Zentralstaaten beziehen sich vor allem auf die Investitionsfinanzierung sowie die Kapazitätsplanung für hochspezialisierte Versorgungsbereiche.

\subsection{Schweden}

Die Kapazitätsplanung für die ambulante und stationäre Versorgung sowie die für Neubau und Erhaltung erforderliche Investitionsfinanzierung ist in Schweden Aufgabe der 18 Provinziallandtage und zwei Regionen. Sie verfügen über alle vier Jahre in unmittelbarer Wahl von der Bevölkerung gewählte Regionalparlamente, die ihrerseits die Leitung (Regierung; schwedisch: styrelse) des Provinziallandtages wählen. Die Provinziallandtage/Regionen und Kommunen haben das Recht, eigene Einkommensteuern zu erheben, die bei den Provinziallandtagen/Regionen zu einem wesentlichen Teil zur Deckung der Gesundheitskosten sowie ebenfalls zur Deckung anfallender Investitionskosten im Gesundheitsbereich verwendet werden. Auf nationaler Ebene werden die Provinziallandtage und Kommunen durch den Verband der schwedischen Kommunen und Provinziallandtage (Sveriges Kommuner och Landtsting - SKL) vertreten.

Die Planung, Vorbereitung und Realisierung von Kapazitätsveränderungen und deren Finanzierung ist Aufgabe der jeweiligen für Gesundheit zuständigen Behörde des Provinziallandtages/ der Region, die von einem durch das Parlament des Provinziallandtages/ der Region gewählten Politiker geleitet wird, der gleichzeitig Mitglied der Provinziallandtags-Regierung ist. In den Parlamenten der Provinziallandtage/Regionen existiert mindestens ein 
für Gesundheitsfragen zuständiger Ausschuss, der die Arbeit der Gesundheitsbehörde begleitet und z.B. politische Initiativen vorbereitet. In den meisten Provinziallandtagen existieren für den Themenbereich Gesundheit aber mehrere Parlamentsausschüsse, im Provinziallandtag von Värmland zum Beispiel ein Ausschuss für Volksgesundheit und Zahnversorgung, ein Krankenhausausschuss sowie ein Ausschuss für die Primär- und Psychiatrieversorgung. Fragen der Kapazitätsplanung und Investitionsfinanzierung werden dort im zuständigen Krankenhausausschuss politisch diskutiert und begleitet. Der Etat der Gesundheitsbehörde, der dann wieder Grundlage für Investitionsvorhaben ist, ist Teil des Gesamtetats des Provinziallandtages/der Region und wird vom jeweiligen Parlament für jeweils ein Jahr beschlossen.

Hochspezialisierte Versorgungsbereiche werden in Schweden traditionell in Kooperation zwischen der nationalen Regierung und den Provinziallandtagen/ Regionen festgelegt in den insgesamt sieben schwedischen Universitäts- bzw. Regionalkrankenhäusern erbracht. Konkret zuständig für die Auswahl und die regionale Zuordnung sowie zeitliche Befristung dieser Zuordnung von hochspezialisierter Versorgung (Rikssjukvård) ist das bei der schwedischen Aufsichtsbehörde für das Gesundheits- und Sozialwesen (Socialstyrelsen) angesiedelte Komitee für spezialisierte medizinische Versorgung (Rikssjukvårdsnämnden), das aus Vertretern der Provinziallandtage/Regionen, des schwedischen Forschungsrates, der schwedischen HealthTechnology-Assessment-Organisation SBU und dem schwedischen Verwaltungsgerichtshof besteht. Vorsitzender des Komitees ist der Generaldirektor von Socialstyrelsen. Die Zuordnungen zu bestimmten Universitäts- bzw. Regionalkrankenhäusern gelten jeweils für fünf Jahre, in manchen Fällen für 5 1/2 Jahre.

Derzeit gelten die folgenden Behandlungen als hochspezialisiert:

- Paediatric heart surgery

- Other paediatric heart surgery

- Ocular oncology

- Treatment of severe burns

- Lung transplantation

- Liver transplantation

- Heart transplantation
- Craniofacial surgery

- Cochlear implantation in infants

- Intrauterine treatments

- Glaucoma in children. ${ }^{4}$

\subsection{Finnland}

Die Kommunen spielen im finnischen Gesundheitssystem eine herausragende Rolle. Sie sind umfassend für die gesundheitliche und soziale Versorgung ihrer Wohnbevölkerung zuständig. Dazu haben sie u.a. das Recht, eine eigene Einkommensteuer zu erheben, aus der der größte Teil der Gesundheitsausgaben finanziert wird. Darüber hinaus erhalten die Kommunen Zuweisungen vom finnischen Zentralstaat, die an demographischen und sozialen Kenndaten orientiert und nicht zweckgebunden sind. Der Zentralstaat erlässt die rechtlichen Rahmenregelungen, nach denen die Kommunen die gesundheitliche Versorgung zu erbringen haben.

$\mathrm{Zu}$ den rechtlichen Rahmenvorschriften gehört u.a., dass jede finnische Kommune Mitglied eines bestimmten Krankenhausbezirkes (sairaanhoitopiiri) sein muss, der für die Versorgung mit ambulanter und stationärer fachärztlicher Versorgung sowie die Notfallversorgung an Akutkrankenhäusern zuständig ist. Insgesamt existieren 20 Krankenhausbezirke, deren Bevölkerung zwischen 45.000 und 1,5 Millionen Menschen variiert. Ein Krankenhausbezirk arbeitet ähnlich einer Holding, zu der mehrere Krankenhäuser gehören. Sie sind jedoch keine eigenen Gebietskörperschaften, sondern eher mit kommunalen Zweckverbänden in der Bundesrepublik Deutschland zu vergleichen. Sie werden von den ihnen angehörenden Kommunen (Beispiel Krankenhausbezirk Turku: 65 Kommunen mit zwischen 160.000 und 220 Einwohnern) getragen und aus diesen heraus politisch gelenkt und verwaltet. Dazu wählen die Gemeinderäte der am Krankenhausbezirk beteiligten Kommunen einen Rat des Krankenhausbezirkes, der seinerseits Lenkung und politische Kontrolle über den Krankenhausbezirk ausübt. Die Gemeinderäte der Kommunen werden alle vier Jahre gewählt; danach werden die Räte der Krankenhausbezirke neu gewählt. Der Rat des jeweiligen Krankenhausbezirkes wählt dann das (hauptamtlich tätige) Management des Krankenhausbezirkes.

Die Krankenhausbezirke sind unmit- telbar sowohl für die Erbringung der ambulanten sowie stationären fachärztlichen Versorgung und Notfallversorgung sowie für deren Kapazitätsplanung und Investitionsfinanzierung zuständig. Die jeweilige Jahresplanung, die auch mögliche Kapazitätsänderungen oder erweiterungen sowie die dazu erforderliche Finanzierungsplanung beinhaltet, muss dem Rat des Krankenhausbezirks zur Abstimmung vorgelegt werden. Der finnische Zentralstaat greift in die Krankenhausplanung der Krankenhausbezirke nur durch die Auslobung von Fördergeldern für bestimmte politisch gewollte Projekte ein. Wenn ein Krankenhausbezirk diese Fördergelder nutzen will, muss er die damit verbundenen Bedingungen erfüllen. Solche Fördergelder wurden zum Beispiel im Zusammenhang mit der landesweiten Einführung einer elektronischen Patientenakte genutzt, um dieses Projekt zu fördern. Für Kapazitätserweiterungen dagegen wird das Förderinstrumentarium so gut wie nicht genutzt.

Insgesamt gibt es in Finnland drei Ebenen der Krankenhausversorgung. Die oberste Ebene der stationären Maximalversorgung stellen fünf Universitätskliniken dar, die gleichzeitig als Zentralkrankenhäuser ihrer Krankenhausbezirke fungieren. Zusätzlich übernehmen sie als Krankenhäuser der Maximalversorgung bestimmte medizinische Aufgaben entweder für einen Teil Finnlands oder aber für das gesamte Land. Auch die übrigen 15 Krankenhausbezirke verfügen jeweils über ein eigenes Zentralkrankenhaus, das allerdings nicht über so viele Spezialitäten und Subspezialitäten verfügt wie die fünf Universitätsklinika. Welche hochspezialisierten Versorgungsbereiche an den fünf Universitätskliniken bzw. den Zentralkrankenhäusern der Krankenhausbezirke angesiedelt werden, wird in einem Komitee beraten und beschlossen, das vom finnischen Gesundheitsministerium eingesetzt worden ist und dem sowohl Vertreter der Krankenhausbezirke als auch der verschiedenen medizinischen Spezialitäten angehören. Unterhalb dieser mittleren Versorgungsebene existieren weitere Regionalkrankenhäuser, üblicherweise mehrere je Krankenhausbezirk, mit Abteilungen für Innere

4 http://www.socialstyrelsen.se/nationalspecialisedmedicalcare 
Medizin und Chirurgie. So existieren etwa im Krankenhausbezirk Turku neben dem Universitätsklinikum Turku mit rund 1.100 Betten weitere fünf regionale Krankenhäuser mit insgesamt 666 Betten.

Die Finanzierung der Krankenhausbezirke und ihrer Krankenhäuser ist seit 1993 vielfach auf eine fallweise leistungsbezogene Vergütung überwiegend nach dem DRG-System umgestellt worden. Dabei werden zwischen dem einzelnen Krankenhaus und der einzelnen die Leistungen bestellenden Kommune Preise und Mengen pro DRG-Gruppe jeweils für ein Jahr ausgehandelt; es gibt keine bezirks- oder landesweit gültigen Preise. Mit den Krankenhäusern, die das DRG-System nicht anwenden, werden Vereinbarungen geschlossen, die sowohl die Preise als auch die Mengen der von der Kommune bestellten Leistungen beinhalten. Die Kommunen haben auch das Recht, Leistungen von öffentlichen oder privaten Krankenhäusern außerhalb des Krankenhausbezirks, der die Kommune angehört, zu beziehen. Diese Leistungen müssen dann je nach finanziellem Umfang finnlandweit oder europaweit ausgeschrieben werden.

\subsection{Dänemark}

Die Kapazitätsplanung und Investitionsfinanzierung im Krankenhausbereich war traditionell Aufgabe der Ämter. Seit der Strukturreform 2007 ist sie Aufgabe der neuen fünf Regionen. Prinzipiell sind die Regionen bei der Erfüllung dieser Aufgabe weitgehend unabhängig. Sie stellen dafür jeweils Jahrespläne für die Gesundheitsversorgung in der Region und ihre Finanzierung auf. Allerdings greift der dänische Staat zunehmend durch die Auslobung von Fördergeldern in die Kapazitätsplanung der Regionen ein, um bestimmte, auf gesamtstaatlicher Ebene festgelegte gesundheitliche und strukturelle Ziele zu erreichen, zu denen vor allem eine zunehmende Zentralisierung und Spezialisierung der Krankenhausversorgung gehört. ${ }^{5}$ Basis dieser Förderpolitik sind Abkommen zwischen der dänischen Regierung und den Regionen, in denen die Verwendung der Gelder festlegt wird. In den Jahren 2009/2010 wurden so zum Beispiel 16 Krankenhausprojekte mit einem Fördervolumen von rund 25 Milliarden dänischen Kronen gefördert. Die Regionen mussten sich darin verpflichten, weitere 15 Milliarden DKR zu finanzieren.

Seit der Gebietsreform 2007 muss die Einrichtung von hochspezialisierter medizinischer Behandlung in einem Krankenhaus von Sundhetsstyrelsen, einer Behörde im Zuständigkeitsbereich des dänischen Gesundheitsministeriums, genehmigt werden. Grundlage sind bindende Richtlinien, die von Sundhetssyrelsen erlassen worden sind. Der dadurch in Gang gesetzte Konzentrationsprozess ist noch nicht abgeschlossen, wird aber zu erheblichen Veränderungen in der dänischen Krankenhausstruktur führen. ${ }^{6}$

\subsection{Norwegen}

Am stärksten zentralisiert sind Kapazitätsplanung und Investitionsfinanzierung in Norwegen; dort ist, wie bereits ausgeführt, der norwegischen Zentralstaat Eigentümer der fünf regionalen Gesundheitskonzerne, die als öffentliche Aktiengesellschaften organisiert und die Betreiber der öffentlichen Krankenhäuser in Norwegen sind. Auch die einzelnen Krankenhäuser sind unterhalb der Ebene des regionalen Gesundheitsunternehmens, zu dem sie gehören, ebenfalls als eigenständige rechtliche Einheiten organisiert. Für die Überwachung der Gesundheitskonzerne ist eine dem Gesundheitsministerium untergeordnete Behörde geschaffen worden. Gleichzeitigt hat der Gesundheitsminister in den Gesellschaftsversammlungen als Vertreter des norwegischen Staates als Anteilseigner die Möglichkeit, in grundsätzlichen Fragen zu entscheiden. Für die Privatisierung von öffentlichen Krankenhäusern ist die Zustimmung des norwegischen Parlaments erforderlich. Alle übrigen Entscheidungen werden auf der Grundlage von grundsätzlichen Plänen und Vereinbarungen mit dem Gesundheitsministerium auf der $\mathrm{Ma}$ nagement-Ebene der fünf Gesundheitsunternehmen getroffen. ${ }^{7}$ Dabei beruht vor allem die Investitionsfinanzierung weitgehend auf vom Staat im Zuge der Budgetplanung bewilligten Geldern. Dagegen liegt die Verantwortung für die Organisation der Einrichtungen und auch für die Verwendung von Investitionsmittel allein beim Management und dem jeweiligen Aufsichtsrat des einzelnen Gesundheitsunternehmens. ${ }^{8}$ In der Praxis entwickelt das Management der jeweiligen regionalen Gesundheitskonzerne eine Planung für die Weiterentwicklung der fachärztlichen ambulanten und stationären sowie der Notfall-Versorgung einschließlich der dazu gehörigen Investitionsfinanzierung, der dann der - vom Gesundheitsminister berufene - Aufsichtsrat zustimmen muss. Solche Planungen waren auch Grundlage dafür, dass die Zahl der Krankenhäuser in Norwegen im Jahr nach der Reform, die im Jahre 2002 in Kraft getreten ist, durch Restrukturierungsmaßnahmen der einzelnen Gesundheitskonzerne von 43 auf 31 zurückgegangen ist. ${ }^{9}$ Das Management der regionalen Gesundheitskonzerne hat auch die Möglichkeit, bestimmte Leistungen von privaten Leistungserbringern einzukaufen statt diese in eigenen Einrichtungen erbringen zu lassen. Auch diese Möglichkeit wurde nach der Reform verstärkt genutzt. ${ }^{10}$

Der norwegische Gesundheitsminister hat das Recht, hochspezialisierte Versorgungsbereiche (sog. tertiärer Level) zu definieren und diese bestimmten Krankenhäusern zuzuordnen oder solche Erlaubnisse auch zu widerrufen. Im Jahr 2010 gab es in Norwegen insgesamt 32 solcher Einrichtungen des tertiären Levels (landsfunksjoner), meist an den fünf Universitätskrankenhäusern des Landes. Dazu gehören zum Beispiel Organtransplantationen, viele Krebsbehandlungen oder die spezialisierte Trombozytenimmunologie.

5 Maria Olejaz et. al.: Health Systems in Transition - Denmark Health System Review; Kopenhagen 2012; S. 84

6 ebd.; S. $146 \mathrm{f}$.

7 Jan Roth Johnsen: Health Systems in Transition Norway; Kopenhagen 2006; S. $20 \mathrm{ff}$. und S.135ff.

8 ebd., S.112

9 ebd.; S. 95

10 ebd. 